Informe especial

\title{
Influencia de los glucocorticoides inhalados sobre la densidad mineral ósea y el metabolismo óseo
}

\author{
Fernando D. Saraví, ${ }^{1,2}$ Manuel A. Guirao, ${ }^{1}$ P. Carlos Elías ${ }^{3,4}$ \\ y Pablo J. Guarnieri ${ }^{3}$
}

RESUMEN Los glucocorticoides inhalados (GCI) constituyen hoy un tratamiento de primera linea del asma bronquial. Los efectos sistémicos de los GCI, como la supresión del eje hipotalámicohipofisario-adrenal, son en general menores que los de los glucocorticoides orales. Sin embargo, existe el riesgo de efectos adversos sobre el hueso a largo plazo. El objetivo del presente trabajo fue revisar los datos publicados acerca de los efectos de los GCI sobre los marcadores del metabolismo óseo y la densidad mineral ósea en adultos y en pacientes pediátricos. Aunque los estudios examinados no proporcionan resultados uniformes, en términos generales sugieren que los GCI pueden afectar al metabolismo y a la densidad mineral ósea, en particular 1) cuando se administran a dosis elevadas (más de $400 \mu \mathrm{g} /$ día en niños y más de $800 \mu \mathrm{g} /$ día en adultos); 2) en pacientes pediátricos, en los que también pueden afectar al crecimiento en estatura; 3) en pacientes cuya ingesta de calcio y vitamina $D$ es inadecuada, y 4) en mujeres postmenopáusicas sin terapia de reposición hormonal. En general, a dosis terapéuticamente equivalentes, la beclometasona tiene mayor efecto deletéreo sobre el hueso que la budesonida, y esta mayor que la fluticasona. Además de la precaución obvia de utilizar la menor dosis eficaz, se proponen como medidas preventivas: 1) la adecuada instrucción sobre el uso de los aerosoles; 2) el uso de cámaras de inhalación; 3) el enjuague bucal tras la administración de GCI, y 4) ajustes o suplementos dietéticos para asegurar una adecuada ingesta de calcio y vitamina D.

La administración crónica de glucocorticoides es una causa importante de osteoporosis secundaria (1-3) y, conse-

\footnotetext{
Unidad de Densitometría Ósea, Escuela de Medicina Nuclear, Mendoza, Argentina. Toda la correspondencia debe ser enviada a Fernando D. Saraví a la siguiente dirección postal: Unidad de Densitometría Ósea, Escuela de Medicina Nuclear, Garibaldi 405, Mendoza 5500, Argentina. Teléfono (54 261) 449 4046. Fax (54 261) 438 0232. Correo electrónico: fsaravi@fmed2.uncu.edu.ar

2 Cátedra de Biofísica, Facultad de Ciencias Médicas de la Universidad Nacional de Cuyo, Mendoza, Argentina.

3 Cátedra de Farmacología, Facultad de Ciencias Médicas de la Universidad Nacional de Cuyo, Mendoza, Argentina.

4 Unidad de Evaluación Funcional Respiratoria, Escuela de Medicina Nuclear, Mendoza, Argentina.
}

cuentemente, de fracturas óseas (4). Dosis superiores a $5 \mathrm{mg} /$ día de prednisona o equivalente son capaces de reducir la densidad mineral ósea (5-7). La densidad mineral del hueso trabecular se reduce al cabo de solo 6 meses de administración de dosis de 10 mg/día de prednisona; el efecto es parcialmente reversible al suspender el tratamiento (8). La dosis total acumulada y la duración del tratamiento son factores importantes en la magnitud del efecto adverso sobre el tejido óseo $(2,6)$.

Durante el crecimiento, la velocidad de formación de hueso es superior a la velocidad de resorción y el balance de calcio es positivo. En el adulto joven, cerca de $10 \%$ del hueso se remodela cada año (9). En esta situación la masa ósea se mantiene relativamente constante como resultado del equilibrio dinámico entre la formación y la resorción óseas, procesos que dependen, respectivamente, de la actividad de los osteoblastos y de los osteclastos. A partir de la cuarta o quinta década de la vida, la resorción de hueso predomina sobre la formación, lo que resulta en una pérdida progresiva de masa ósea. Aunque ambos sexos se ven afectados, la declinación de la masa ósea es 
acelerada en la mujer en la década que sigue a la menopausia (10). La reducción de la masa ósea es el principal factor de riesgo de fracturas en personas de edad avanzada (11) y constituye un problema de salud mundial (12).

Los glucocorticoides afectan a la biología ósea tanto por sus efectos directos sobre las células óseas como por sus influencias indirectas sobre las hormonas que regulan a dichas células y al metabolismo del calcio y el fósforo. Bajo la acción de los glucocorticoides, la formación de hueso disminuye debido a la menor proliferación de los precursores osteoblásticos y a la reducción de la actividad de síntesis de los osteoblastos ya existentes. La supresión de la secreción de adrenocorticotropina (ACTH) reduce la producción adrenal de esteroides sexuales y contribuye a la menor actividad anabólica. Por su parte, la resorción ósea aumenta debido al incremento de la actividad osteoclástica, e indirectamente, debido a la mayor secreción de hormona paratiroidea, a la reducción de la secreción de calcitonina y a la disminución de la absorción intestinal y de la reabsorción renal de calcio y fósforo. Por tanto, los glucocorticoides a la vez reducen la formación y aumentan la resorción de hueso, provocando así una disminución de la masa ósea $(1-3,6,13)$. Aunque es posible tratar la osteoporosis inducida por glucocorticoides, es evidente que es preferible prevenirla (1-3, 6, 13-15).

En pacientes asmáticos, el tratamiento crónico con glucocorticoides orales reduce la densidad mineral ósea e incrementa la prevalencia de fracturas (16). Los efectos de los glucocorticoides orales sobre el metabolismo óseo son independientes de su efectividad en el tratamiento del asma (17). Un reciente estudio británico (18) reveló que la mayoría de los pacientes sometidos a tratamiento crónico con glucocorticoides orales no recibía tratamiento preventivo contra la osteoporosis; $19 \%$ eran asmáticos o padecían otra enfermedad obstructiva crónica.

Los glucocorticoides inhalados (GCI) se recomiendan actualmente como tratamiento crónico de primera línea del asma bronquial moderada a grave (19) y sin duda han contribuido a reducir los problemas del tratamiento con corticoides sistémicos (20-22), pero ello no implica que carezcan por completo de impacto sobre el hueso.

Los GCI beclometasona, budesonida, flunisolida, fluticasona y triamcinolona poseen una elevada potencia farmacológica, y solo cerca de $10 \%$ de la dosis inhalada alcanza el pulmón; la mayor parte es deglutida y absorbida en el tracto digestivo. Aunque la budesonida y la flunisolida sufren una alta extracción y metabolización hepática, y la fluticasona presenta baja biodisponibilidad por vía oral $(19,20)$, una fracción significativa de la dosis puede alcanzar la circulación sistémica. Existen pruebas de que los GCI afectan al eje hipotalámico-hipofisario-adrenal tanto en adultos (23-24) como en niños (25). Existen varias revisiones sobre los efectos adversos sistémicos de los GCI (26-29).

La preocupación por los posibles efectos óseos de los GCI está particularmente justificada en mujeres postmenopáusicas, que de por sí tienen mayor riesgo de osteoporosis $(10,12)$, en los pacientes que requieren tratamiento crónico con altas dosis de GCI (30), y en los niños, debido a que se hallan en el proceso de crecimiento óseo (31). En el presente trabajo, se revisan los estudios publicados durante la última década acerca de los efectos de los GCI sobre los marcadores del metabolismo óseo y la densidad mineral ósea, identificados mediante una búsqueda en la base de datos Medline.

\section{ESTRATEGIAS DE VALORACIÓN DE LOS EFECTOS DE LOS GCI SOBRE EL HUESO}

La estimación de los efectos de los GCI sobre el tejido óseo se ha realizado mayormente mediante dos modalidades mutuamente complementarias. La más directa es la determinación de la densidad y el contenido mineral del hueso, generalmente mediante absorciometría de rayos $X$ de energía dual (DEXA) o tomografía computadorizada cuantitativa. Ambas técnicas son acep- tablemente precisas y reproducibles $(32,33)$. Los datos densitométricos son obviamente importantes, dado que la densidad mineral ósea es el principal determinante del riesgo de fracturas $(11,34,35)$. Sin embargo, las modificaciones de la densidad mineral ósea son relativamente lentas. Por esta razón, se emplea asimismo una variedad de análisis bioquímicos que, en principio, permiten detectar precozmente cambios sutiles del metabolismo óseo. Los denominados marcadores óseos (cuadro 1) son básicamente moléculas cuya concentración en suero $\mathrm{u}$ orina guarda relación con la velocidad de formación o de resorción de la matriz extracelular (36, 37). La excreción urinaria de calcio e hidroxiprolina son indicadores poco sensibles de la resorción ósea que se han utilizado en los estudios más antiguos.

Se requiere cautela al interpretar los resultados de estudios basados en estas determinaciones bioquímicas, en particular cuando los marcadores de formación y de resorción varían en el mismo sentido. Por ejemplo, un aumento simultáneo de ambos tipos de marcadores refleja una mayor velocidad de recambio pero, por sí mismo, no permite anticipar el efecto sobre la masa ósea.

Un factor de confusión importante en los estudios clínicos es el tratamiento previo con corticosteroides orales o la necesidad de emplear tratamientos breves con corticosteroides orales para controlar la sintomatología.

\section{ESTUDIOS EN PACIENTES PEDIÁTRICOS}

En tratamientos breves que compararon el efecto de los glucocorticoides orales con el de la budesonida, esta última no modificó los marcadores de formación o de resorción ósea en pacientes pediátricos de 1 a 3 años (38) y mayores (39). El tratamiento con dosis elevadas de beclometasona (1 500 $\mu \mathrm{g} /$ día) durante 6 semanas redujo la osteocalcina y el propéptido carboxiterminal del procolágeno de tipo I (PICP) sin afectar al telopéptido carboxiterminal del colágeno de tipo I (ICTP) ni a la desoxipiridinolina, en 
CUADRO 1. Principales indicadores del metabolismo óseo empleados en estudios recientes sobre el efecto de los glucocorticoides inhalados sobre el hueso

\begin{tabular}{|c|c|c|c|}
\hline Marcador & Sinónimo (o acrónimo) & Determinado en & Comentarios \\
\hline Fosfatasa alcalina ósea & (BAP, BALP) & Suero & $\begin{array}{l}\text { Aproximadamente } 50 \% \text { de la actividad de fosfatasa alcalina sérica } \\
\text { total; mejor medida mediante anticuerpos monoclonales }\end{array}$ \\
\hline Osteocalcina & Proteína ósea Gla, (BGP) & Suero & $\begin{array}{l}\text { Proteína no colágena más abundante de la matriz ósea. Posee } 49 \\
\text { aminoácidos, con tres residuos de ácido } \gamma \text {-carboxiglutámico (Gla). } \\
\text { El suero contiene la proteína completa y diversos fragmentos }\end{array}$ \\
\hline $\begin{array}{l}\text { Propéptido carboxiterminal } \\
\text { del procolágeno tipo I }\end{array}$ & (PICP) & Suero & $\begin{array}{l}\text { Fragmento peptídico liberado durante el procesamiento } \\
\text { postranslacional del procolágeno }\end{array}$ \\
\hline \multicolumn{4}{|l|}{ Resorción ósea } \\
\hline $\begin{array}{l}\text { Fosfatasa ácida resistente al } \\
\text { tartarato }\end{array}$ & (TRAP) & Suero & Isoenzima osteoclástica \\
\hline Piridinolina & $\begin{array}{l}\text { Hidroxilisilpiridinolina } \\
\text { (Pyr) }\end{array}$ & Orina & $\begin{array}{l}\text { Molécula "puente" que liga lateralmente las moléculas de colágeno } \\
\text { entre sí }\end{array}$ \\
\hline
\end{tabular}

tanto que una dosis equipotente de fluticasona (750 $\mu \mathrm{g} /$ día ) no afectó a ninguno de los marcadores (40). Sin embargo, en otro estudio (41) que comparó el efecto de dosis de 800 $\mu \mathrm{g} /$ día de beclometasona y budesonida se verificó que no afectaron a la ostecalcina, si bien ambos GCI redujeron un marcador de formación (PICP) y otro de resorción (piridinolinas urinarias). En un estudio (42), la beclometasona administrada durante seis meses o más no modificó la osteocalcina (aunque el nivel basal fue menor en los pacientes asmáticos), pero redujo la fosfatasa ácida resistente al tartarato, un marcador de resorción. Igualmente, en un estudio prospectivo de 5 meses de duración (43), el tratamiento con budesonida redujo los marcadores de formación ósea osteocalcina y PICP, pero los mismos autores comprobaron posteriormente (44) que este GCI también reducía los marcadores de resorción ICTP y desoxipiridinolina. En conjunto, estos datos sugieren que el tratamiento con GCI reduce la velocidad de recambio óseo en pacientes pediátricos, lo cual ha sido confirmado recientemente por otros investigadores (45).

Si los procesos de formación y resorción ósea se reducen proporcionalmente, es posible que los GCI no afecten a la mineralización. Kinberg et al. (46) no hallaron diferencias entre la densidad mineral ósea de 97 niños normales y la de 30 pacientes asmáticos sometidos a tratamiento con corticoides orales, GCI o cromoglicato sódico. Comparaciones transversales en pacientes asmáticos no han mostrado reducciones de la densidad mineral ósea ni del contenido mineral óseo lumbar asociadas al empleo de beclometasona a dosis de 200 a $450 \mu \mathrm{g} /$ día (47-49). König et al. (42) no hallaron diferencias de mineralización en el radio, la columna lumbar ni el esqueleto completo en grupos relativamente pequeños (8 a 12 pacientes) tratados con beclometasona (400 a $800 \mu \mathrm{g} /$ día durante no menos de 6 meses) en comparación con niños sanos. Otros investigadores $(47,50-53)$ han comparado la variación en la mineralización ósea en niños asmáticos tratados con GCI durante 6 meses o más sin hallar dife- rencias significativas en la velocidad de incremento de la densidad mineral. Sin embargo, un estudio (54) de 40 pacientes que recibieron GCI durante 3 a 8 años mostró, en comparación con 148 niños sanos de la misma población, una reducción de la densidad mineral de la totalidad del organismo que se correlacionó inversamente con la duración del tratamiento; en cambio la densidad mineral lumbar no se vio afectada. Agertoft y Pedersen (55) estudiaron 268 pacientes asmáticos, de los cuales 157 recibieron budesonida a una dosis media de $504 \mu \mathrm{g} /$ día durante 3 a 6 años, y no hallaron diferencias en la densidad mineral o el contenido mineral de la totalidad del organismo, ni en la composición corporal en comparación con los otros 111 niños que nunca habían recibido corticosteroides exógenos durante más de 14 días; tampoco hubo correlación entre los parámetros citados y la duración del tratamiento o la dosis actual o acumulada de budesonida.

La evaluación de los posibles efectos de los GCI sobre el crecimiento se ve dificultada por una serie de posibles 
factores de confusión tanto genéticos como ambientales que pueden explicar la existencia de resultados conflictivos. Algunos estudios (47, 51, 53, 56-58) no han hallado efectos adversos de los GCI sobre la talla o la velocidad de aumento de la talla. En cambio, Saha et al. (59) estudiaron 201 pacientes prepuberales durante varios años y hallaron una reducción de la velocidad de crecimiento que no mostró relación con la dosis de GCI y fue mayor durante el primer año de tratamiento. En otra investigación prospectiva (60) de cuatro años de duración en 3347 niños (2 355 tratados con GCI) se observaron menores tallas y velocidades de crecimiento en los pacientes tratados con dosis de GCI $\geq 400 \mu \mathrm{g} /$ día, aunque el efecto fue modesto.

Entre los diversos GCI, la beclometasona es la que se ha asociado más reiteradamente con el retraso del crecimiento (61-66). Por término medio, la reducción de la velocidad de crecimiento fue de $1 \mathrm{~cm} /$ año. El retraso del crecimiento no parece deberse a alteraciones del eje hipotalámico-hipofisarioadrenal ni de la secreción de somatotropina (67). La budesonida también puede reducir la velocidad de crecimiento, al menos en algunos pacientes $(45,67)$, aunque el efecto es menos manifiesto. De hecho, no pudo demostrarse dicha influencia en dos investigaciones bien controladas con un número importante de pacientes prepuberales (68) y adolescentes (69). Tampoco se han observado efectos adversos de la fluticasona sobre el crecimiento $(70,71)$, excepto a dosis más altas que las usuales (72).

La mayor parte de los estudios citados han sido a medio plazo (1 a 5 años). Los datos sobre el efecto de los GCI sobre la talla final de los pacientes asmáticos son, por razones obvias, menos abundantes. Los dos estudios publicados $(56,73)$ sugieren que los GCI no afectan a la talla alcanzada en la edad adulta. Aunque todavía no está claro si existe un efecto sobre la talla final adulta, un grupo de expertos de la Administración de Alimentos y Medicamentos (Food and Drug Administration) de los Estados Unidos de América recomendó en 1998 que los medicamentos que incluyan GCI lle- ven una advertencia indicando que "pueden causar una reducción de la velocidad de crecimiento cuando se administran a pacientes pediátricos" (74).

\section{ESTUDIOS EN ADULTOS}

En voluntarios sanos, el efecto más constante de los GCI es la reducción de la osteocalcina sérica, cuya magnitud guarda relación con la dosis (75-79). Una dosis diaria de budesonida de $3200 \mu \mathrm{g}$ posee un efecto depresor de la osteocalcina similar al de $40 \mathrm{mg}$ de prednisolona (80). El uso de una cámara de inhalación suprime la reducción de la osteocalcina observada con dosis altas (2 $000 \mu \mathrm{g})$ de beclometasona (81). A esa dosis, la beclometasona, pero no la budesonida, reduce la fosfatasa alcalina sérica y aumenta la excreción urinaria de hidroxiprolina (82). Con budesonida a dosis de 2400 $\mu \mathrm{g} /$ día tampoco se observaron efectos sobre las concentraciones séricas de hormona paratiroidea y de metabolitos activos de la vitamina $\mathrm{D}$ ni sobre la excreción urinaria de calcio y fósforo (83).

En pacientes asmáticos tratados con GCI solos o en combinación con glucocorticoides orales no se alteró la concentración de hormona paratiroidea ni la absorción intestinal de calcio, pero hubo mayor número de fracturas vertebrales en el grupo tratado con terapia combinada $(14 / 25 ; 56 \%)$ que en los que recibieron solo GCI $(2 / 25 ; 8 \%)$ (84). Los estudios a corto plazo en pacientes asmáticos (85-87) muestran, como en voluntarios sanos, una reducción de la osteocalcina sérica. El efecto de la beclometasona sobre la osteocalcina es mayor que el de la fluticasona a dosis de igual eficacia terapéutica (88, 89), aunque dosis altas de fluticasona también reducen la osteocalcina (90). La influencia de los GCI sobre otros indicadores del metabolismo óseo es menos clara, especialmente para los marcadores de formación, ya que se han descrito tanto aumentos (87) como disminuciones $(88,91)$ y ausencia de cambios (85). Lo mismo ocurre con el marcador de resorción ICTP, que dis- minuyó en un estudio (91), mientras que no se modificó en otros $(87,88)$. Existe la posibilidad de que el patrón sérico y urinario de marcadores óseos se modifique a lo largo del tratamiento; al cabo de dos años y medio de tratamiento, Kerstjens et al. (87) solamente hallaron una reducción del ICTP sérico.

En varias investigaciones (92-100), dosis pequeñas a moderadas de beclometasona o budesonida (300 a 1000 $\mu \mathrm{g}$ /día) administradas durante 1 a 10 años no han tenido efectos adversos sobre la densidad mineral medida en la columna lumbar, el fémur proximal y el antebrazo. Sin embargo, en otros estudios $(99,100)$ la reducción de la densidad mineral lumbar mostró correlación con la dosis diaria. La fluticasona no modificó los marcadores óseos ni la densidad mineral lumbar a dosis bajas (101) ni altas (102). Al igual que en pacientes pediátricos, la fluticasona parece tener menor influencia sobre el hueso que la beclometasona a dosis igualmente eficaces desde el punto de vista terapéutico (103).

Otros estudios indican que los GCI pueden reducir la densidad mineral ósea. En algunos casos la interpretación es difícil debido a la administración previa o simultánea de glucocorticoides orales $(104,105)$. Se ha informado de un caso de fracturas vertebrales atribuible al empleo de GCI (106). Mujeres postmenopáusicas a las que se les administraron GCI tuvieron densidades minerales intermedias entre las que no los recibían y las tratadas con glucocorticoides orales (107). Es posible que los GCI afecten diferencialmente a la densidad mineral en diversos sitios. Lau et al. (108) compararon la densidad mineral de 144 pacientes (106 tratados con GCI) con controles normales y verificaron que los pacientes tratados de ambos sexos tenían menor densidad mineral lumbar, pero las mujeres sufrieron, además, una reducción de la densidad mineral del esqueleto completo, y los varones una reducción de la densidad mineral del fémur proximal. La menor densidad mineral lumbar y femoral en el estudio de Ip et al. (109) se debió al grupo de mujeres tratadas. Existe una 
correlación negativa entre la densidad mineral y la dosis diaria de GCI (109, 110). En un estudio transversal de pacientes que recibieron altas dosis de GCI (111) se separaron los que anteriormente habían recibido tratamiento oral de los que no lo habían recibido; los pacientes de ambos grupos mostraron reducciones comparables de la densidad mineral lumbar y del fémur proximal; los marcadores de formación estaban reducidos, en tanto que los de resorción eran normales. Dosis intermedias de beclometasona o budesonida redujeron la osteocalcina y la densidad mineral del fémur proximal, pero no la de la columna lumbar, al cabo de 1 año; la magnitud de la reducción de la densidad mineral se correlacionó con la duración del tratamiento (112). En otro estudio (113) la influencia de la beclometasona sobre la densidad mineral fue mayor que la de la budesonida. Egan et al. (114) registraron en pacientes tratados con GCI una densidad mineral basal inferior a la de sujetos normales; tras 2 años de tratamiento con dosis altas, hubo cierta reducción de la densidad mineral en el grupo tratado con beclometasona, pero no en el tratado con fluticasona.

Un aspecto de considerable interés práctico es que el tabaquismo y la dieta baja en calcio acentúan el efecto deletéreo de los GCI en el fémur y la columna, respectivamente (108), en tanto que los suplementos de calcio (1 g/día) contrarrestan dicho efecto (115). Otro tanto puede decirse del tratamiento de reposición con estrógenos en mujeres postmenopáusicas (110).

\section{CONCLUSIONES Y RECOMENDACIONES}

Algunos estudios acerca del efecto de los GCI sobre el hueso tienen características que dificultan su interpretación, como la administración previa o simultánea de glucocorticoides orales y muestras relativamente pequeñas y no siempre homogéneas. Además, su duración ha sido en la mayoría de los casos breve a intermedia. Hacen faltan más estudios longitudinales a largo plazo y con gran número de pacientes, los cuales presentan obvias dificultades para su realización.

De todos modos, los datos disponibles indican que los GCI tienen mucho menor acción sobre el hueso que dosis de glucocorticoides orales que producen el mismo efecto terapéutico; no obstante, dicha acción puede ser significativa, en particular con dosis altas. Entre los GCI, la beclometasona parece ser la que tiene mayor impacto sobre el hueso, seguida de la budesonida y la fluticasona.

Los pacientes que requieren altas dosis de GCI deben ser evaluados en cuanto a su estado mineral. Los nuevos marcadores pueden tener un papel modesto en la evaluación del efecto sobre el metabolismo óseo, solo como indicadores que no sustituyen la determinación de la densidad mineral, $\mathrm{y}$, en niños, la velocidad de crecimiento y la talla final. Debido a que los GCI pueden reducir el recambio óseo sin afectar a la mineralización si las velocidades de formación y de resorción continúan acopladas, al emplear marcadores es necesario deter- minar al menos uno de formación y otro de resorción. A diferencia de lo que ocurre en adultos, en la población pediátrica la osteocalcina no es un buen indicador.

La dosis diaria de GCI podría ser más importante que la dosis total acumulada o la duración del tratamiento como determinante de los efectos adversos sobre el hueso. Existe consenso de que se pueden administrar sin serio riesgo dosis diarias de beclometasona y budesonida de hasta $400 \mu \mathrm{g}$ en niños y de hasta $1600 \mu \mathrm{g}$ en adultos, y de fluticasona de hasta 200 y $500 \mu \mathrm{g}$, respectivamente $(20,116)$.

Los pacientes deben ser entrenados en el correcto uso de los aerosoles (117). El empleo de cámaras de inhalación reduce la cantidad de GCI que se absorbe en las mucosas o se ingiere (81, 117-119). El enjuague bucal tiene un efecto similar (119). Es posible que, análogamente a lo observado en pacientes con artritis reumatoide tratados con glucocorticoides orales a bajas dosis (120), los suplementos de calcio y vitamina $\mathrm{D}$ tengan un efecto protector; en todo caso, debe recomendarse una ingesta adecuada de ambos, en especial durante la niñez $(115,121)$. Finalmente, en mujeres postmenopáusicas la terapia de reposición de estrógenos parece contrarrestar eficazmente los efectos adversos de los GCI (110). Como, pese a la aparición de nuevos y promisorios agentes antiasmáticos (122), es probable que se sigan empleando los GCI en el tratamiento crónico del asma, se justifican las precauciones para reducir al mínimo sus posibles efectos sobre el hueso.

\section{REFERENCIAS}

1. Gulko PS, Mulloy AL. Glucocorticoidinduced osteoporosis: pathogenesis, prevention and treatment. Clin Exp Rheumatol 1996;14:199-206.

2. Sambrook PN. Corticosteroid induced osteoporosis. J Rheumatol 1996;23(supl 45):S19S22.

3. Adachi JD. Corticosteroid-induced osteoporosis. Am J Med Sci 1997;313:41-49.

4. Lems WF, Jahangier ZN, Jacobs JWG, Bijlsma JWJ. Vertebral fractures in patients with rheumatoid arthritis treated with corticosteroids. Clin Exp Rheumatol 1995;13: 293-297.

5. Buckley LM, Leib ES, Cartularo KS, Vacek PM, Cooper SM. Effects of low dose corticosteroids on the bone mineral density of patients with rheumatoid arthritis. J Rheumatol 1995; 22:1055-1059.

6. Dequeker J, Westhovens R. Low dose corticosteroid associated osteoporosis in rheumatoid arthritis and its prophylaxis and treat- ment: bones of contention [Editorial]. J Rheumatol 1995;22:1013-1019.

7. Saito JK, Davis JW, Wasnich RD, Ross PD. Users of low-dose glucocorticoids have increased bone loss rates: a longitudinal study. Calcif Tissue Int 1995;57:115-119.

8. Laan RFJM, van Riel PLCM, van de Putte LBA, van Erning LJTO, van't Hof MA, Lemmens JAM. Low-dose prednisolone induces rapid reversible axial bone loss in patients with rheumatoid arthritis: a randomized, 
controlled study. Ann Intern Med 1993;119: 963-968.

9. Peel N, Eastell R. ABC of rheumatology: osteoporosis. BMJ 1995;310:989-992.

10. National Osteoporosis Foundation. Physician's guide to prevention and treatment of osteoporosis. Washington: National Osteoporosis Foundation; 1998.

11. Melton LJ III, Thamer M, Ray NF, Chan JK, Chesnut CH III, Einhorn TA, et al. Fractures attributable to osteoporosis: report from the National Osteoporosis Foundation. J Bone Miner Res 1997;12:16-23.

12. Melton LJ. Global aspects of osteoporosis epidemiology. En: Papapoulos SE, Lips P, Pols HAP, Johnston CC, Delmas PD, eds. Osteoporosis 1996. Amsterdam: Elsevier; 1996; 79-86.

13. Canalis E. Mechanisms of glucocorticoid action in bone: implications to glucocorticoidinduced osteoporosis. J Clin Endocrinol Metab 1996;81:3441-3447.

14. Adler RA. Preventing and treating glucocorticoid osteoporosis. En: Rosen CJ, ed. Osteoporosis: diagnostic and therapeutic principles. Totowa: Humana Press; 1996:239-249.

15. Eastell R. Management of corticosteroidinduced osteoporosis: UK Consensus Group Meeting on Osteoporosis. J Intern Med 1995; 237:439-447.

16. Adinoff AD, Hollister JR. Steroid-induced fractures and bone loss in patients with asthma. N Eng J Med 1983;309:265-268.

17. Lane SJ, Vaja S, Swaminathan R, Lee TH. Effects of prednisolone on bone turnover in patients with corticosteroid resistant asthma. Clin Exp Allergy 1996;26:1197-1201.

18. Walsh LJ, Wong CA, Pringle M, Tattersfield AE. Use of oral corticosteroids in the community and the prevention of secondary osteoporosis: a cross sectional study. BMJ 1996; 313:344-346.

19. Expert Panel Report 2. Guidelines for the diagnosis and management of asthma. Washington: National Institutes of Health; 1997. (Publication 97-4051).

20. Barnes PJ. Inhaled glucocorticoids for asthma. N Eng J Med 1995;332:868-875.

21. Barnes PJ. Corticosteroids. En: O'Byrne PM, Thomson NC, eds. Manual of asthma. London: W.B. Saunders; 1995:219-253.

22. Cockcroft DW, Kalra S. Outpatient asthma management. Med Clin North Am 1996;80: 701-718.

23. Wihl JA, Andersson KE, Johansson SA. Systemic effects of two nasally administered glucocorticosteroids. Allergy 1997;52:620626.

24. Grahnen A, Jansson B, Brundin RM, LingAndersson A, Lonnebo A, Johansson M, et al. A dose-response study comparing suppression of plasma cortisol induced by fluticasone propionate from Diskhaler and budesonide from Turbuhaler. Eur J Clin Pharmacol 1997;52:261-267.

25. Pedersen S, Fuglsang G. Urine cortisol excretion in children treated with high doses of inhaled corticosteroids: a comparison of budesonide and beclomethasone. Eur Respir J 1988;1:433-435.

26. Hanania NA, Chapman KR, Kesten S. Adverse effects of inhaled corticosteroids. Am J Med 1995;98:196-208.
27. Ahmann AJ. Endocrine-metabolic effects of asthma treatment. Immunol Allergy Clin North Am 1997;17:701-726.

28. Barnes N. Relative safety and efficacy of inhaled corticosteroids. J Allergy Clin Immunol 1998;101:S460-S464.

29. Lipsworth BJ. Systemic adverse effects of inhaled corticosteroid therapy. A systematic review and meta-analysis. Arch Intern Med 1999;159:941-955.

30. Toogood JH. High-dose inhaled steroid therapy for asthma. J Allergy Clin Immunol 1989;83:528-536.

31. Wagener JS, Wojtczack HA. Inhaled steroids in children: Risks versus rewards [Editorial]. J Pediatr 1998;132:381-383.

32. Wahner HW, Fogelman I. The evaluation of osteoporosis: dual energy X-ray absorptiometry in clinical practice. London: Martin Dunitz; 1994.

33. Swedish Council on Technology Assessment in Health Care. Bone density measurement. A systematic review. J Intern Med 1997; 241(supl 739):12-22.

34. Miller PD, McClung M. Prediction of fracture risk. I: Bone density. Am J Med Sci 1996; 312:257-259.

35. Marshall D, Johnell O, Wedel H. Meta-analysis of how well measures of bone mineral density predict occurrence of osteoporotic fractures. BMJ 1996;312:1254-1259.

36. Calvo MS, Eyre DR, Gundberg CM. Molecular basis and clinical applications of biological markers of bone turnover. Endocr Rev 1996;17:333-368.

37. Garnero P, Delmas PD. Les marqueurs biochimiques du remodelage osseux: application a l'exploration de ostéoporoses. Ann Biol Clin (Paris) 1999;57:137-148.

38. Hedlin G, Svedmyr J, Ryden AC. Systemic effects of a short course of bethametasone compared with high-dose inhaled budesonide in early childhood asthma. Acta Paediatr 1999;88:48-51.

39. Wolthers OD, Riis BJ, Pedersen S. Bone turnover in asthmatic children treated with oral prednisolone or inhaled budesonide. Pediatr Pulmonol 1993;16:341-346.

40. Bootsma GP, Dekhuijzen PN, Festen J, Mulder PG, Swinkels LM, van Herwaarden CL. Fluticasone propionate does not influence bone metabolism in contrast to beclomethasone dipropionate. Am J Respir Crit Care Med 1996;153:924-930.

41. Birkebaek NH, Esberg G, Andersen K, Wolthers $\mathrm{O}$, Hassager $\mathrm{C}$. Bone and collagen turnover during treatment with inhaled dry powder budesonide and beclomethasone dipropionate. Arch Dis Child 1995;73:524- 527.

42. König P, Hillman L, Cervantes C, Levine C, Maloney C, Douglass B, et al. Bone metabolism in children with asthma treated with inhaled beclomethasone dipropionate. J Pediatr 1993;122:219-226.

43. Sorva R, Turpeinen M, Juntunen-Backman $\mathrm{K}$, Karonen SL, Sorva A. Effects of inhaled budesonide on serum markers of bone metabolism in children with asthma. J Allergy Clin Immunol 1992;90:808-815.

44. Sorva R, Tähtela R, Turpeinen M, JuntunenBackman K, Haahtela T, Risteli L, et al. Changes in bone markers in children with asthma during inhaled budesonide and ne- docromil treatments. Acta Paediatr 1996; 85:1176-1180.

45. Crowley S, Trivedi P, Risteli L, Risteli J, Hindmarsh PC, Brook CG. Collagen metabolism and growth in prepubertal children with asthma treated with inhaled steroids. J Pediatr 1998;132:409-413.

46. Kinberg KA, Hopp RJ, Biven RE, Gallagher GC. Bone mineral density in normal and asthmatic children. J Allergy Clin Immunol 1994;94:490-497.

47. Baraldi E, Bollini MC, De Marchi A, Zacchello F. Effect of beclomethasone dipropionate on bone mineral content assessed by X-ray densitometry in asthmatic children: a longitudinal evaluation. Eur Respir J 1994; 7:710-714

48. Hopp RJ, Degan JA, Phelan J, Lappe J, Gallagher GC. Cross-sectional study of bone density in asthmatic children. Pediatr Pulmonol 1995;20:189-192.

49. Martinati LC, Bertoldo F, Gasperi E, Micelli $\mathrm{S}$, Boner AL. Effect on cortical and trabecular bone mass of different antiinflammatory treatments in preadolescent children with chronic asthma. Am J Respir Crit Care Med 1996;153:232-236.

50. Hopp RJ, Degan JA, Biven RE, Kinberg K, Gallagher GC. Longitudinal assessment of bone mineral density in children with chronic asthma. Ann Allergy Asthma Immunol 1995; 75:143-148.

51. Martinati LC, Bertoldo F, Gasperi E, Fortunati $\mathrm{P}$, Lo Cascio V, Boner AL. Longitudinal evaluation of bone mass in asthmatic children treated with inhaled beclomethasone dipropionate or cromolyn sodium. Allergy 1998;53:705-708.

52. Gregson RK, Rao R, Murrills AJ, Taylor PA, Warner JO. Effect of inhaled corticosteroids on bone mineral density in childhood asthma: comparison of fluticasone propionate with beclomethasone dipropionate. Osteoporos Int 1998;8:418-422.

53. Chay OM, Goh A, Lim WH, Leong KH, Lou J. Effects of inhaled corticosteroid on bone turnover in children with bronchial asthma. Respirology 1999;4:63-67.

54. Boot AM, de Jongste JC, Verberne AA, Pols HA, de Muinck Keizer-Schrama SM. Bone mineral density and bone metabolism of prepubertal children with asthma after longterm treatment with inhaled corticosteroids. Pediatr Pulmonol 1997;24:379-384.

55. Agertoft L, Pedersen S. Bone mineral density in children with asthma receiving long-term treatment with inhaled budesonide. Am J Respir Crit Care Med 1998;157:178-183.

56. Balfour-Lynn L. Growth and childhood asthma. Arch Dis Child 1986;61:1049-1055.

57. Ninan TK, Russell G. Asthma, inhaled corticosteroid treatment, and growth. Arch Dis Child 1992;67:703-705.

58. Allen DB, Mullen M, Mullen B. A metaanalysis of the effect of oral and inhaled corticosteroids on growth. J Allergy Clin Immunol 1994;93:967-976.

59. Saha MT, Laippala P, Lenko HL. Growth of asthmatic children is slower during than before treatment with inhaled glucocorticoids. Acta Paediatr 1997;86:138-142.

60. McCowan C, Neville RG, Thomas GE, Crombie IK, Clark RA, Ricketts JW, et al. Effect of 
asthma and its treatment on growth: four year follow up of cohort of children from general practices in Tayside, Scotland. BMJ 1998;316:668-672.

61. Nassif E, Weinberger M, Sherman B, Brown K. Extrapulmonary effects of maintenance corticosteroid therapy with alternate-day prednisone and inhaled beclomethasone in children with chronic asthma. J Allergy Clin Immunol 1987;80:518-529.

62. Littlewood JM, Johnson AW, Edwards PA, Littlewood AE. Growth retardation in asthmatic children treated with inhaled beclomethasone dipropionate. Lancet 1988;1 (8577):115-116.

63. Tinkelman DG, Reed CE, Nelson HS, Offord KP. Aerosol beclomethasone dipropionate compared with theophylline as primary treatment of chronic, mild to moderately severe asthma in children. Pediatrics 1993;92: 64-77.

64. Doull I, Freezer N, Holgate S. Osteocalcin, growth, and inhaled corticosteroids: a prospective study. Arch Dis Child 1996;74: 497-501.

65. Simons FER. A comparison of beclomethasone, salmeterol and placebo in children with asthma. N Eng J Med 1997;337:16591665.

66. Veberne AAPH, Frost C, Roorda RJ, van der Laag H, Kerrebijn KF. One-year treatment with salmeterol compared with beclomethasone in children with asthma. The Dutch Paediatric Asthma Study Group. Am J Respir Crit Care Med 1997;156:688-695.

67. Crowley S, Hindmarsh PC, Matthews DR, Brook CG. Growth and the growth hormone axis in prepubertal children with asthma. J Pediatr 1995;126:297-303.

68. Agertoft L, Pedersen S. Effects of long-term treatment with an inhaled corticosteroid on growth and pulmonary function in asthmatic children. Respir Med 1994;88:373-381.

69. Merkus PJ, van Essen-Zandvliet EE, Duiverman EJ, van Houwelingen HC, Kerrebijn KF, Quanjer PH. Long-term effect of inhaled corticosteroids on growth rate in adolescents with asthma. Pediatrics 1993;91:1121-1126.

70. Price JF, Russel G, Hindmarsh PC, Weller P, Heaf DP, Williams J. Growth during one year of treatment with fluticasone propionate or sodium cromoglycate in children with asthma. Pediatr Pulmonol 1997;24:178186.

71. Allen DB, Bronsky EA, LaForce CF, Nathan RA, Tinkelman DG, Vandewalker ML, et al. Growth in asthmatic children treated with fluticasone propionate. J Pediatr 1998;132: 472-477.

72. Todd G, Dunlop K, McNaboe J, Ryan MF, Carson D, Shields MD. Growth and adrenal suppression in asthmatic children treated with high-dose fluticasone propionate. Lancet 1996;348:27-29.

73. Silverstein MD, Yungiger JW, Reed CE, Petterson T, Zimmerman D, Li JT, et al. Attained adult height after childhood asthma: effect of glucocorticoid therapy. J Allergy Clin Immunol 1997;99:466-474.

74. Food and Drug Administration. Class labeling for intranasal and orally administered corticosteroid containing drug products regarding the potential for growth suppression in children. [Sitio en Internet] FDA Talk Paper. Disponible en: http://www.fda.gov/cder/ news/cs-label.htm. Acceso el 8 marzo, 2000.

75. Jennings $\mathrm{BH}$, Anderson KE, Johansson SA. Assessment of systemic effects of inhaled glucocorticosteroids: comparison of the effects of inhaled budesonide and oral prednisolone on adrenal function and markers of bone turnover. Eur J Clin Pharmacol 1991; 40:77-82.

76. Teelucksingh S, Padfield PL, Tibi L, Gough $\mathrm{KJ}$, Holt PR. Inhaled corticosteroids, bone formation, and osteocalcin. Lancet 1991;338: 60-61.

77. Toogood JH, Jennings B, Hodsman AB, Baskerville J, Fraher LJ. Effects of dose and dosing schedule of inhaled budesonide on bone turnover. J Allergy Clin Immunol 1991; 88:572-580.

78. Pouw EM, Prummel MF, Oosting H, Roos $\mathrm{CM}$, Endert E. Beclomethasone inhalation decreases serum osteocalcin concentrations. BMJ 1991;302:627-628.

79. Meeran K, Hattersley A, Burrin J, Shiner R, Ibbertson K. Oral and inhaled corticosteroids reduce bone formation as shown by plasma osteocalcin levels. Am J Resp Crit Care Med 1995;151:333-336.

80. Hodsman AB, Toogood JH, Jennings B, Fraher LJ, Baskerville JC. Differential effects of inhaled budesonide and oral prednisolone on serum osteocalcin. J Clin Endocrinol Metab 1991;72:530-540.

81. Brown PH, Matusiewicz SP, Shearing C, Tibi L, Greening AP, Crompton GK. Systemic effects of high dose inhaled steroids: comparison of beclomethasone dipropionate and budesonide in healthy subjects. Thorax 1993;48:967-973

82. Ali NJ, Capewell S, Ward MJ. Bone turnover during high dose inhaled corticosteroid treatment. Thorax 1991;46:160-164.

83. Toogood JH, Crilly RG, Jones G, Nadeau J, Wells GA. Effect of high dose inhaled budesonide on calcium and phosphate metabolism and the risk of osteoporosis. Am Rev Respir Dis 1988;138:57-61.

84. Luengo M, Picado C, Piera C, Guanabens N, Montserrat JM, Rivera J, et al. Intestinal calcium absorption and parathyroid hormone secretion in asthmatic patients on prolonged oral or inhaled steroid treatment. Eur Respir J 1991;4:441-444.

85. Puolijoki H, Lippo K, Herrala J, Salmi J, Tala E. Inhaled beclomethasone decreases serum osteocalcin in postmenopausal asthmatic women. Bone 1992;13:285-288.

86. Leech JA, Hodder RV, Ooi DS, Gay J. Effects of short-term inhaled budesonide and beclomethasone dipropionate on serum osteocalcin in premenopausal women. Am Rev Respir Dis 1993;148:113-115.

87. Kerstjens HA, Postma DS, van Doormaal JJ, van Zanten AK, Brand PL, Dekhuijzen PN, et al. Effects of short-term and long-term treatment with inhaled corticosteroids on bone metabolism in patients with airways obstruction. Dutch CNSLD Study Group. Tho$\operatorname{rax} 1994 ; 49: 652-656$

88. Bootsma GP, Dekhuijzen PN, Festen J, Mulder PG, Swinkels LM, van Herwaarden CL. Fluticasone propionate does not influence bone metabolism in contrast to beclometha- sone dipropionate. Am J Respir Crit Care Med 1996;153:924-930.

89. Malo JL, Cartier A, Ghezzo H, Mark S, Brown J, Laviolette $\mathrm{M}$, et al. Skin bruising adrenal function and markers of bone metabolism in asthmatics using inhaled beclomethasone and fluticasone. Eur Respir J 1999;13:993-998.

90. Lipworth BJ, Clark DJ. High dose inhaled steroids in asthmatic children [Carta]. Lancet 1996;348:820.

91. Haapasaari K, Rossi O, Risteli J, Oikarinen A. Effects of long-term inhaled corticosteroids on skin collagen synthesis and thickness in asthmatic patients. Eur Respir J 1998;11: 139- 143.

92. Wolff AH, Adelsberg B, Aloia J, Zitt M. Effect of inhaled corticosteroid on bone density in asthmatic patients: a pilot study. Ann Allergy 1991;61:117-121.

93. Luengo M, Del Río L, Guanabens N, Picado C. Long-term effect of oral and inhaled glucocorticoids on bone mass in chronic asthma. A two year follow-up study. Eur Respir J 1991;4 (supl 14):S342.

94. Herrala J, Puolijoki H, Impivaara O, Liippo $\mathrm{K}$, Tala E, Nieminen MM. Bone mineral density in asthmatic women on high-dose inhaled beclomethasone dipropionate. Bone 1994;15:621-623.

95. Luengo M, del Rio L, Pons F, Picado C. Bone mineral density in asthmatic patients treated with inhaled corticosteroids: a case-contro study. Eur Respir J 1997;10:2110-2113.

96. Gagnon L, Boulet LP, Brown J, Desrosiers T. Influence of inhaled corticosteroids and dietary intake on bone density and metabolism in patients with moderate to severe asthma. J Am Diet Assoc 1997;97:1401-1406.

97. Boulet LP, Giguere MC, Milot J, Brown J. Effects of long-term use of high-dose inhaled steroids on bone density and calcium metabolism. J Allergy Clin Immunol 1994;94:796-803.

98. Hughes JA, Conry BG, Male SM, Eastell R. One year prospective open study of the effect of high dose inhaled steroids, fluticasone propionate, and budesonide on bone markers and bone mineral density. Thorax 1999; $54: 223-229$

99. Wisniewski AF, Lewis SA, Green DJ, Maslanka W, Burrell $\mathrm{H}$, Tattersfield AE. Cross sectional investigation of the effects of inhaled corticosteroids on bone density and bone metabolism in patients with asthma. Thorax 1997;52:853-860.

100. Boulet LP, Milot J, Gagnon L, Poubelle PE, Brown J. Long-term influence of inhaled corticosteroids on bone metabolism and density. Are biological markers predictors of bone loss? Am J Respir Crit Care Med 1999; 159: 838-844

101. Howland WC III. Fluticasone dipropionate: topical or systemic effects? Clin Exp Allergy 1996;26(supl 3):18-22.

102. Li JT, Ford LB, Chervinsky P, Weisberg SC, Kellerman DJ, Faulkner KG, et al. Fluticasone propionate powder and lack of clinically significant effects on hypothalamicpituitary-adrenal axis and bone mineral density over 2 years in adults with mild asthma. J Allergy Clin Immunol 1999;103:1062-1068.

103. Pauwels RA, Yernault JC, Demedts MG, Geusens P. Safety and efficacy of fluticasone 
and beclomethasone in moderate to severe asthma. Belgian Multicenter Study Group. Am J Respir Crit Care Med 1998;157:827-832.

104. Packe GE, Douglas JG, McDonald AF, Robins SP, Reid DM. Bone density in asthmatic patients taking high dose inhaled beclomethasone dipropionate and intermittent systemic corticosteroids. Thorax 1992;47: 414-417.

105. Packe GE, Robb O, Robins SP, Reid DM, Douglas JG. Bone density in asthmatic patients taking inhaled corticosteroids: comparison of budesonide and beclomethasone dipropionate. J R Coll Phys Lond 1996;30: 128-132.

106. Laroche M, Porteau L, Caron P, Moulinier L, Didier A, Amigues JM, et al. Osteovertebral fractures in a man under high-dose inhaled glucocorticoid therapy. Rev Rhum Eng Ed 1997;64:267-270.

107. Marystone JF, Barrett-Connor EL, Morton DJ. Inhaled and oral corticosteroids: their effects on bone mineral density in older adults. Am J Public Health 1995;85:1693-1695.

108. Lau EM, Li M, Woo J, Lai C. Bone mineral density and body composition in patients with airflow obstruction-the role of inhaled steroid therapy, disease and lifestyle. Clin Exp Allergy 1998;28:1066-1071.

109. Ip M, Lam K, Yam L, Kung A, Ng M. Decreased bone mineral density in premenopausal asthma patients receiving longterm inhaled steroids. Chest 1994;105:17221727.
110. Toogood JH, Baskerville JC, Markov AE, Hodsman AB, Fraher LJ, Jennings $B$, et al. Bone mineral density and the risk of fracture in patients receiving long-term inhaled steroid therapy for asthma. J Allergy Clin Immunol 1995;96:157-166.

111. Ebeling PR, Erbas B, Hopper JL, Wark JD, Rubinfeld AR. Bone mineral density and bone turnover in asthmatics treated with long-term inhaled or oral glucocorticoids. J Bone Miner Res 1998;13:1283-1289.

112. Hanania NA, Chapman KR, Sturtridge WC, Szalai JP, Kesten S. Dose-related decrease in bone density among asthmatic patients treated with inhaled corticosteroids. J Allergy Clin Immunol 1995;96:571-579.

113. Struijs A, Mulder $H$. The effects of inhaled glucocorticoids on bone mass and biochemical markers of bone homeostasis: a 1-year study of beclomethasone versus budesonide. Neth J Med 197;50:233-237.

114. Egan JJ, Maden C, Kalra S, Adams JE, Eastell $\mathrm{R}$, Woodcock AA. A randomized, doubleblind study comparing the effects of beclomethasone and fluticasone on bone density over two years. Eur Respir J 1999;13: 1267-1275.

115. Wang WQ, Ip MS, Tsang KW, Lam KS. Antiresorptive therapy in asthmatic patients receiving high-dose inhaled steroids: a prospective study for 18 months. J Allergy Clin Immunol 1998;101:445-450.

116. Jackson LD, Polygenis D, McIvor RA, Worthington I. Comparative efficacy and safety of inhaled corticosteroids in asthma. Can J Clin Pharmacol 1999;6:26-37.

117. Li JTC, Reed CE. Proper use of aerosol corticosteroids to control asthma. Mayo Clin Proc 1989;64:205-210.

118. Brown PH, Blundell G, Greening AP, Crompton GK. Do large volume spacer devices reduce the systemic effects of high dose inhaled corticosteroids? Thorax 1990;45:736-739.

119. Selroos O, Halme M. Effect of a volumatic spacer and mouth rinsing on systemic absorption of inhaled corticosteroids from a metered dose inhaler and dry powder inhaler. Thorax 1991;46:891-894.

120. Buckley LM, Leib ES, Cartularo KS, Vacek PM, Cooper SM. Calcium and vitamin $\mathrm{D}_{3}$ supplementation prevents bone loss in the spine secondary to low-dose corticosteroids in patients with rheumatoid arthritis. A randomized, double-blind, placebo-controlled trial. Ann Intern Med 1996;125:961-968.

121. Martin AD, Bailey DA, McKay HA, Whiting $\mathrm{S}$. Bone mineral and calcium accretion during puberty. Am J Clin Nutr 1997;66:611-615.

122. Duplantier AJ, Turner CR. Novel pharmacological approaches to the treatment of asthma: status and potential of therapeutic classes. Drug Discov Today 1996;1:199-208.

Manuscrito recibido el 10 de diciembre de 1997 y aceptado para publicación, tras revisión, el 22 de octubre de 1999.

ABSTRACT Inhaled glucocorticoids (IGs) are today the first-line treatment for bronchial asthma. The systemic effects of inhaled glucocorticoids, such as suppressing the hypothalamic-pituitary-adrenal axis, are generally less than those with oral glucocorticoids. However, there is a long-term risk of adverse effects on bone. The objective of this piece was to review the published data on the effects of IGs on bone metabolism markers and bone mineral density in adults and in pediatric patients. The reviewed studies do not provide uniform results. Nevertheless, in general they suggest that IGs can affect metabolism and bone mineral density, especially: 1) when high doses are administered (more than $400 \mu \mathrm{g} /$ day in children and more than $800 \mu \mathrm{g} /$ day in adults), 2) in pediatric patients, in whom growth in stature can also be affected, 3) in patients whose intake of calcium and vitamin $D$ is inadequate, and 4) in postmenopausal women not undergoing hormone replacement therapy. In general, at therapeutically equivalent doses, beclomethasone has a greater deleterious effect on bone than does budesonide, which in turn has more of an effect than does fluticasone. In addition to the obvious precaution of using the lowest effective dose, other proposed preventive measures include: 1) adequate instruction on the use of aerosols, 2 ) the use of large volume spacer devices, 3) rinsing the mouth after administering IGs, and 4) dietary adjustments or supplements in order to ensure an adequate intake of calcium and vitamin D. 